\title{
Wilson Disease in Children - a Rare but Treatable Autosomal Recessive Metabolic Disorder
}

\author{
${ }^{1}$ Mani Kant Kumar, ${ }^{2}$ Ramanuj Singh, ${ }^{3}$ Ashutosh Anand, ${ }^{4}$ Sujeet Kumar \\ ${ }^{\prime}$ Associate Professor, Department of Pediatrics, \\ ${ }^{2}$ Assistant Professor, Department of Anatomy, \\ ${ }^{3}$ 3Intern Student ${ }^{, 4}$ Assistant Professor, Department of Otorhinolaryngology, Narayan Medical College and \\ Hospital, Jamuhar, Sasaram, Bihar, India.
}

\begin{abstract}
Wilson's disease (WD) is a rare autosomal recessive inborn error of copper metabolism caused by a mutation to the copper-transporting gene ATP7B. The presentation is usually neurologic or hepatic, seen in $40 \%$ of patients. Diagnosis depends primarily on clinical features, biochemical parameters and the presence of the Kayser-Fleischer ring. Here a case of Wilson disease with various neurological manifestations without any clinical involvement of the liver and clinical presentation, diagnosis and treatment is discussed.
\end{abstract}

Key Words: ATP7B, Dystonia, Kayser-Fleischer ring, Wilson Disease,

\section{Introduction}

Wilson's disease (WD) is an inherited disorder of copper metabolism. The condition was first defined by Dr Samuel Alexander Kinnier Wilson in 1912, also known as hepatolenticular degeneration ${ }^{[1]}$. Wilson's disease (WD) is a rare autosomal recessive inborn error of copper metabolism caused by a mutation to the copper-transporting gene ATP7B. The WD gene, ATP7B, is located on chromosome 13q14.3. The incidence of WD is estimated to be 1 in 30,000 individuals and carrier frequency is approximately 1 in $90^{[2]}$. The ATP7B gene, encodes a metal-transporting P-type adenosine triphosphatase (ATPase), which is expressed mainly in hepatocytes and functions in the transmembrane transport of copper within hepatocytes. Absent or reduced function of ATP7B protein leads to decreased hepatocellular excretion of copper into bile. This results in hepatic copper accumulation and injury. Eventually, copper is released into the bloodstream and deposited in other organs, notably the brain, kidneys, and cornea. Failure to incorporate copper into ceruloplasmin is an additional consequence of the loss of functional ATP7B protein. The hepatic production and secretion of the ceruloplasmin protein without copper, apoceruloplasmin, result in the decreased blood level of ceruloplasmin found in most patients with WD due to the reduced half-life of apoceruloplasmin. ${ }^{[3]}$. The manifestations are more likely to be hepatic in early childhood and neurological in adolescents ${ }^{[4]}$. Neurological manifestations of Wilson disease can vary extremely and often diagnosed with long delay. Here a case of Wilson disease with Neurological manifestations and treatment of the Wilson Disease are to be discussed.

A 14 years old Hindu boy youngest of 4 siblings, a product of non-consanguineous marriage, presented with pain in right knee joint and difficulty in walking for 4 months, 10 months back, which was treated by many physician without any significant improvement. After 4 months of onset of the pain, he developed abnormal movement of the right upper and lower limb in the form of repetitive tonic movement, which was progressive and involved all four limbs and for last 4 months he was non ambulatory. Parent consulted many physician and neurologist for these problems and diagnosis was missed and so no improvement, and then brought to our institute. This abnormal movement subsided during sleep. Patient also had progressive dysarthria. His Elder brother also had history of very slowly progressive abnormal movement and dysarthria. There was no history of measles during early childhood and convulsion. His developmental milestones were normal. On examination his vital signs were normal. There was no pallor, icterus or significant lymphadenopathy. Nervous system examination revealed dystonia, exaggerated deep tendon reflexes, ankle clonus and positive Babinski's sign. Muscle power was $>4 / 5$ in all limbs. Ophthalmoscopic examination by ophthalmologist by slit lamp showed Kayser- Fleischer ring (K-F ring) on both sides. Other systemic examination did not reveal any abnormality. 


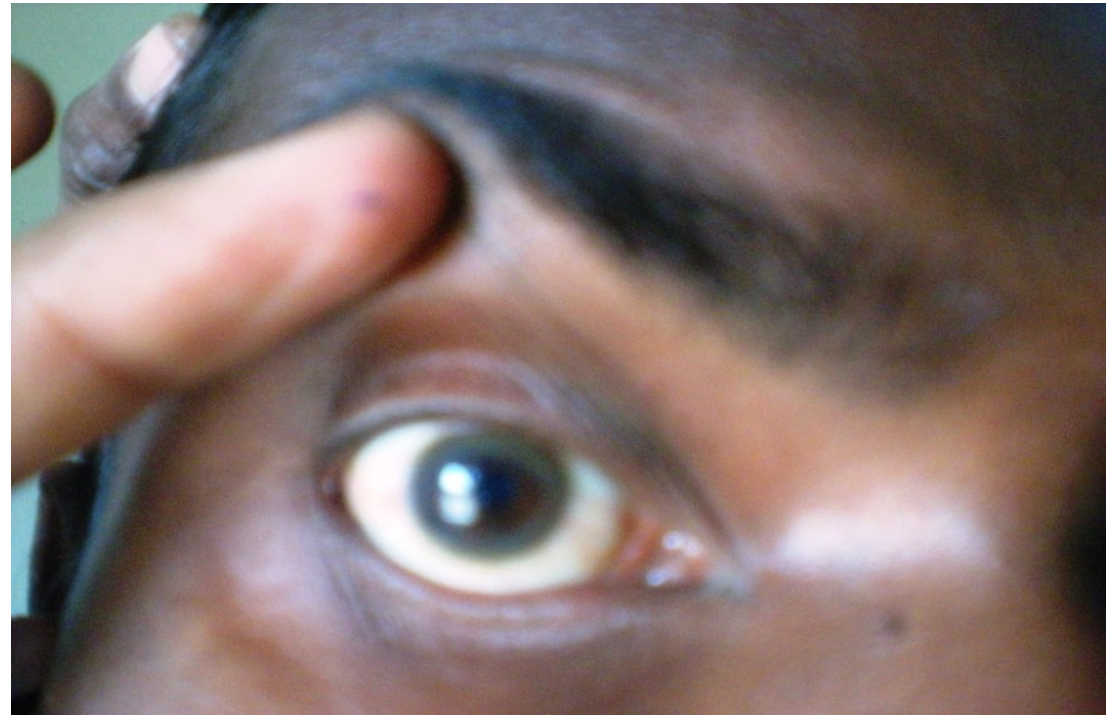

Figure1:The Kayser-Fleischer ring around the periphery of the cornea caused by deposition of copper in Descemet's membrane.

Complete blood count revealed haemoglobin $10 \mathrm{gm} / \mathrm{dl}$, total leukocytes count 5600/cumm (Neutrophil $62 \%$, Lymphocytes 33\%, Monocytes $2 \%$ and eosinophils 3\%) and platelets was 340,000/cumm. Serum Electrolytes and renal function were normal. Total Serum bilirubin was $0.6 \mathrm{mg} / \mathrm{dl}$ (Direct bilirubin $0.2 \mathrm{mg} / \mathrm{dl}$ ) and total serum protein was $6.8 \mathrm{gm} / \mathrm{dl}$ (Albumin $4.2 \mathrm{gm} / \mathrm{dl}$ ), serum transaminases (AST, ALT) and alkaline phosphatise (ALP) were 38, 24, and $168 \mathrm{IU} / \mathrm{L}$ respectively. His prothrombin time (PT) and activated partial thromboplastin time (aPTT) were within normal limits. His serum ceruloplasmin was $95 \mathrm{mg} / \mathrm{L}$ (Normal 180$350 \mathrm{mg} / \mathrm{L}$ ) and 24 hours urine copper excretion was $160 \mathrm{mg}$. Urine routine and microscopic examination. Ultrasonography of the abdomen was normal.

On magnetic resonance imaging (MRI) in Wilson diasease-T2-weighted axial MRI demonstrates the "face of the giant panda" in the midbrain with high signal in tegmentum and normal red nuclei (arrow) (Figure 2) .

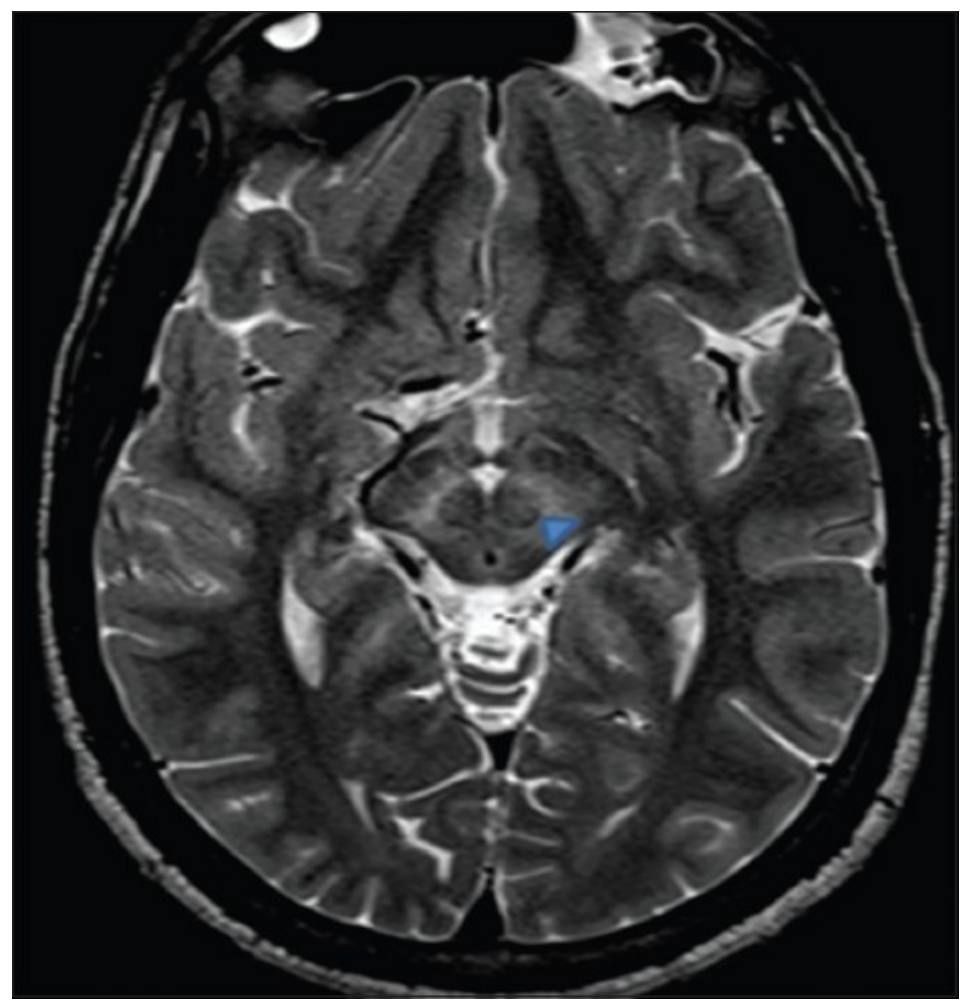

Figure 2 : T2-Weighted axial sequence showing typical "Face of the Giant panda" in the midbrain with high signal in tegmentum and normal red nuclei (arrow) . 
Diagnosis of Wilson disease with neurological manifestation was made on the basis of clinical presentation, K-F ring in the both eyes, low serum ceruloplasmin, increased 24 hours urinary copper excretion and Typical MRI findings. Parents were explained regarding prognosis and available treatment in our country. Patient was started on oral zinc (as zinc acetate) at dose of $1 \mathrm{mg} / \mathrm{kg} / \mathrm{dose}$ of elemental zinc 8 hourly and Trihexiphenidyl to control dystonia. He was advised to avoid food with high copper content such as chocolate, nuts, legumes, mushrooms, shellfish and liver. Parents were also advised to avoid use copper utensils in the household for storage of water, cooking food. After two weeks of treatment, his dystonia had reduced in intensity.

\section{Discussion:}

Wilson's disease (WD) is a rare autosomal recessive inborn error of copper metabolism caused by a mutation to the copper-transporting gene ATP7B. The WD gene, ATP7B, is located on chromosome 13q14.3. The incidence of WD is estimated to be 1 in 30,000 individuals and carrier frequency is approximately 1 in 90 ${ }^{[2]}$. The ATP7B gene, encodes a metal-transporting P-type adenosine triphosphatase (ATPase), which is expressed mainly in hepatocytes and functions in the transmembrane transport of copper within hepatocytes (figure 1).

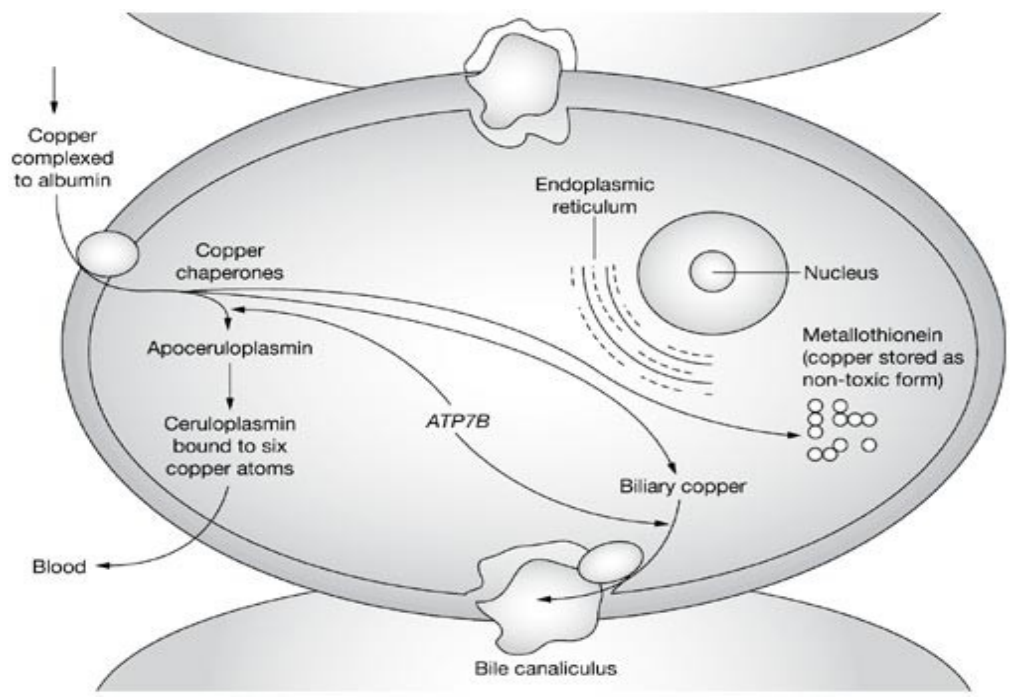

Figure 3: Schematic representation of copper metabolism within a liver cell.

Absent or reduced function of ATP7B protein leads to decreased hepatocellular excretion of copper into bile. Copper first accumulates in the liver; hence the usual presentation during childhood is that of hepatic disease. After the liver storage capacity for copper gets saturated, copper gets redistributed with accumulation in the nervous system, cornea, kidneys and other organs ${ }^{[5]}$. Most patients present in the second decade of life with a primary hepatic presentation with the remainder of patients presenting during the third and fourth decade with a primarily neurologic or psychiatric presentation ${ }^{[6]}$.

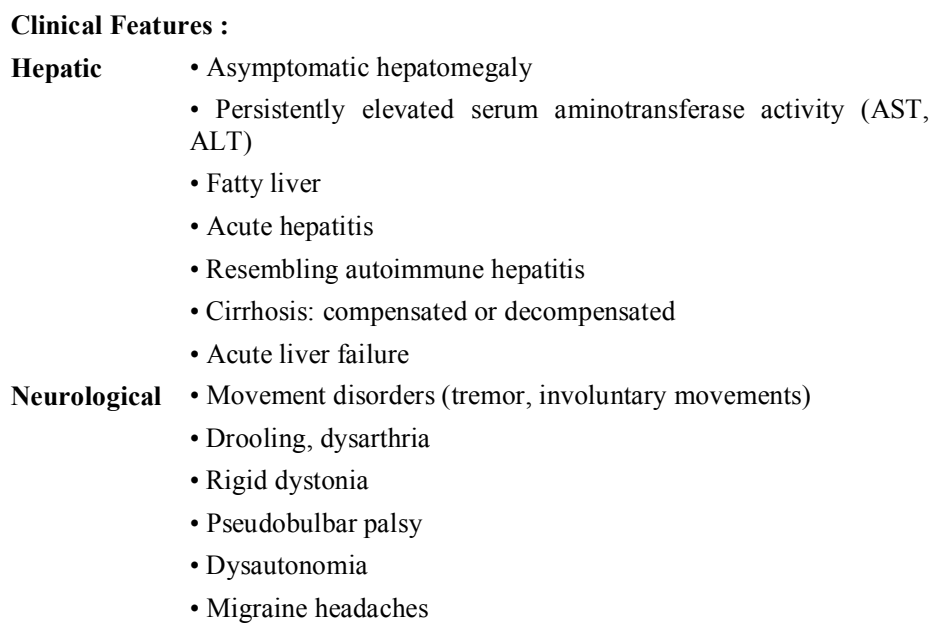




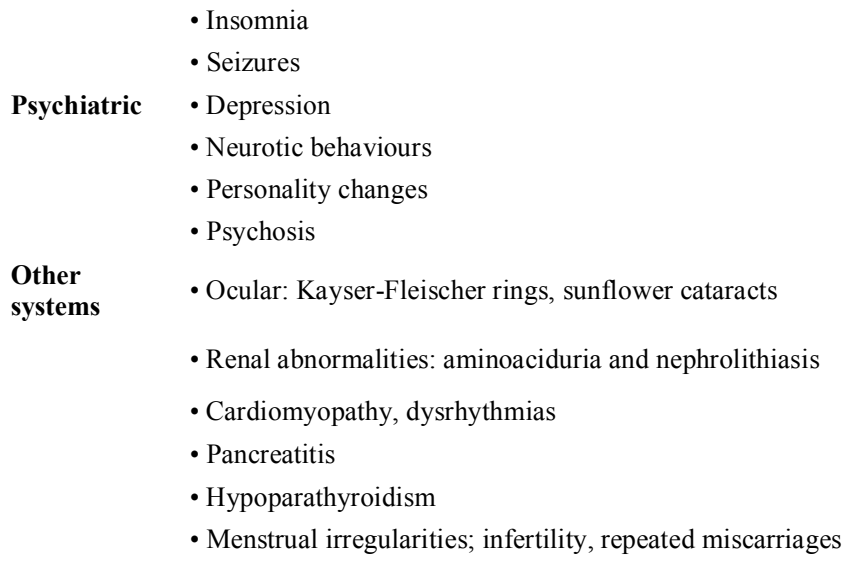

Diagnosis: Diagnostic tests for Wilson disease are listed in Table 1. Serum ceruloplasmin levels should not be used for definitive diagnosis because they are normal in up to $10 \%$ of affected patients and are reduced in $20 \%$ of carriers. Kayser-Fleischer rings (figure 2) can only be diagnosed definitively by an ophthalmologist using slit lamp.

Urine copper is an important diagnostic tool but must be collected carefully to avoid contamination. An estimation of 24-hour urinary copper excretion is another reliable test for confirmation of WD. Normal excretion is between 20 and $50 \mu \mathrm{g}$ per day; in cases of $\mathrm{WD}$, excretion is increased to in excess of $100 \mu \mathrm{g}$ per day.

The "Gold standard" for diagnosis remains liver biopsy with quantitative copper assays. Affected patients have values $>200 \mu \mathrm{g} / \mathrm{gm}$ dry weight of liver. Copper stains are not reliable.

Table 1- Useful Tests for Wilson Disease ${ }^{[10]}$

\begin{tabular}{|c|c|c|c|c|}
\hline Test & Usefulness $^{\mathrm{a}}$ & Normal Value & $\begin{array}{l}\text { Heterozygous } \\
\text { Carriers }\end{array}$ & Wilson Disease \\
\hline $\begin{array}{l}\text { Serum } \\
\text { ceruloplasmin }\end{array}$ & + & $\begin{array}{l}180-350 \mathrm{mg} / \mathrm{L} \\
(18-35 \mathrm{mg} / \mathrm{dL})\end{array}$ & Low in $20 \%$ & Low in $90 \%$ \\
\hline KF rings & ++ & Absent & Absent & $\begin{array}{l}\text { Present in } 99 \%+\text { if neurologic or } \\
\text { psychiatric symptoms present } \\
\text { Present in } 30-50 \% \text { in hepatic } \\
\text { presentation and presymptomatic } \\
\text { state }\end{array}$ \\
\hline 24-h urine $\mathrm{Cu}$ & +++ & $\begin{array}{l}0.3-0.8 \\
\operatorname{mol}(20-50 \\
\qquad \mathrm{g})\end{array}$ & $\begin{array}{l}\text { Normal to } 1.3 \\
\text { mol }(80\end{array}$ & $\begin{array}{l}>1.6 \quad \operatorname{mol}(>100 \quad \mathrm{~g}) \text { in } \\
\text { symptomatic patients } \\
0.9 \text { to }>1.6 \quad \mathrm{~mol}(60 \text { to }>100 \\
\mathrm{g}) \text { in presymptomatic patients }\end{array}$ \\
\hline Liver $\mathrm{Cu}$ & ++++ & $\begin{array}{l}0.3-0.8 \\
\mathrm{~mol} / \mathrm{g}(20-50 \\
\quad \mathrm{g}) \text { tissue }\end{array}$ & $\begin{array}{l}\text { Normal to } 2.0 \\
\text { mol }(125\end{array}$ & $\begin{array}{l}>3.1 \quad \text { mol }(>200 \quad \mathrm{~g}) \\
\text { (obstructive liver disease can cause } \\
\text { false-positive results) }\end{array}$ \\
\hline $\begin{array}{l}\text { Haplotype } \\
\text { analysis }\end{array}$ & $\begin{array}{l}++++ \\
\text { (Siblings } \\
\text { only) }\end{array}$ & 0 Matches & 1 Match & 2 Matches \\
\hline
\end{tabular}

${ }^{a}$ Usefulness: +, somewhat useful, to ++++, very useful.

\section{Neuroimaging}

Neuroimaging plays an important role in both the diagnosis of WD and the monitoring of patients during therapy. Magnetic Resonance Imaging (MRI) is the Neuroimaging of choice. On T1-weighted images, generalized brain atrophy is seen in three-quarters of cases, and hypointensities in the basal ganglia in two-thirds 
of cases. On T2-weighted images, one-third of cases demonstrate hyperintensity in the basal ganglia, white matter, thalamus or brainstem . These abnormalities are caused by neuronal loss, gliosis, degeneration of fibers, and vacuolization associated with increased water content in the brain. Typical 'face of the giant panda', which is seen in T2-weighted images of the midbrain and 'face of the miniature panda', which can be seen in the tegmentum region of the pons in the same sequence (11). The original description of the 'face of the giant panda' sign by Hitoshi et al (12) consisted of high signal intensity in tegmentum except for red nucleus, preservation of signal intensity of the lateral portion of the pars reticulata of the substantia nigra and hypointensity of the superior colliculus. Signal abnormalities vary according to the stage of the disease, and can be reversible with therapy in the early stages.

\section{Genetic investigation:}

Molecular genetic studies are becoming available for clinical use. Pedigree analysis using haplotypes based on polymorphisms surrounding the WD gene is commercially available from specific clinical laboratories. The use of mutation screening to identify defects in the ATP7B gene can provide unequivocal confirmation of WD in an affected symptomatic or presymptomatic individual. Progress in the diagnosis of WD has been made by studying microsatellite markers flanking the ATP7B gene and using linkage analysis to elucidate disease transmission in siblings of affected individuals ${ }^{[15]}$. Marker-based diagnosis should be undertaken when the specific defect in the ATP7B gene is not known.

With a diagnosis of WD, it is mandatory to counsel family members on the importance of biochemical or genetic screening of siblings and other family members, to identify those who might be presymptomatic gene mutation carriers. Monitoring of the presymptomatic individual and placement on a treatment regimen before the onset of clinical symptoms can then be carried out as appropriate. The probability of family members of an affected individual being similarly affected (carrying two mutated genes) is $25 \%$ for siblings and $0.5 \%$ for offspring ${ }^{[16]}$.

\section{Treatment:}

The first step in evaluating patients presenting with hepatic decompensation is to establish disease severity, which can be assessed using the Nazar prognostic index (Table 2). Patients with scores $<7$ can usually be managed with medical therapy.

The aim of medical treatment for WD is to remove the toxic deposit of copper from the body to produce a negative copper balance, and to prevent its reaccumulation. Successful therapy is measured in terms of a restoration of normal levels of free serum copper and its excretion in the urine.

Recommended anticopper treatments are listed in Table 3. Penicillamine was previously the primary anticopper treatment but now plays a minor role because of its toxicity and because it often worsens existing neurologic disease if used as initial therapy. If penicillamine is given, it should always be accompanied by $25 \mathrm{mg} / \mathrm{d}$ of pyridoxine. Trientine is a less toxic chelator and is supplanting penicillamine when a chelator is indicated.

Side effects from D-penicillamine can occur both early and late in the treatment period. Early side effects include a hypersensitivity reaction characterized by fever, skin rash and lymphadenopathy that commonly occurs within 3 weeks of commencing medication. In $20-30 \%$ of cases, an exacerbation of the neurological symptoms occurs over a period of 2 weeks to 12 months (commonly after 6 weeks) ${ }^{[17]}$.

Trientine ${ }^{[18]}$ and ammonium tetrathiomolybdate ${ }^{[19]}$ are considered to be safer alternatives to D-penicillamine. Trientine is a less potent copper remover than D-penicillamine, and its toxic profile is similar to that of Dpenicillamine, although side effects are less frequent and generally milder. Ammonium tetrathiomolybdate, an agent previously used to treat copper toxicosis in animals, has been advocated because of its lower toxic profile, but it is still an experimental drug that is not routinely available, and its long-term safety and efficacy is unknown.

For patients with hepatitis or cirrhosis, but without evidence of hepatic decompensation or neurologic/psychiatric symptoms, zinc is the therapy of choice, although some advocate therapy with trientine. Zinc has proven efficacy in Wilson disease and is essentially nontoxic. It produces a negative copper balance by blocking intestinal absorption of copper, and it induces hepatic metallothionein synthesis, which sequesters additional toxic copper. All presymptomatic patients should be treated prophylactically, since the disease is close to $100 \%$ penetrant. 
Table 2: Prognostic Index of Nazer ${ }^{[10]}$

\begin{tabular}{|c|c|c|c|c|c|c|}
\hline \multirow[t]{2}{*}{ Laboratory Measurement } & \multirow[b]{2}{*}{ Normal Value } & \multicolumn{5}{|c|}{ Score (in Points) } \\
\hline & & $\mathbf{0}$ & 1 & 2 & 3 & 4 \\
\hline Serum bilirubin ${ }^{\mathrm{a}}$ & $0.2-1.2 \mathrm{mg} / \mathrm{dL}$ & $<5.8$ & $5.8-8.8$ & $8.8-11.7$ & $11.7-17.5$ & $>17.5$ \\
\hline Serum aspartate transferase (AST) & $10-35 \mathrm{IU} / \mathrm{L}$ & $<100$ & $100-150$ & $151-200$ & $201-300$ & $>300$ \\
\hline Prolongation of prothrombin time (seconds) & - & $<4$ & $4-8$ & $9-12$ & $13-20$ & $>20$ \\
\hline
\end{tabular}

${ }^{\mathrm{a}}$ If hemolysis is present, the serum bilirubin cannot be used as a measure of liver function until the hemolysis subsides.

Table 3: Recommended Anticopper Drugs for Wilson Disease ${ }^{[10]}$

\begin{tabular}{|l|l|l|}
\hline Disease Status & First Choice & Second Choice \\
\hline Initial hepatic & & \\
\hline Hepatitis or cirrhosis without decompensation & Zinc $^{\mathrm{a}}$ & Trientine \\
\hline Hepatic decompensation & & Penicillamine $^{\mathrm{b}}$ and zinc \\
\hline Mild & Trientine ${ }^{\mathrm{b}}$ and zinc & Hepatic transplantation \\
\hline Moderate & Trientine and zinc & Trientine and zinc \\
\hline Severe & Hepatic transplantation & Zinc \\
\hline Initial neurologic/psychiatric & Tetrathiomolybdate ${ }^{\mathrm{a}}$ and zinc & Trientine \\
\hline Maintenance & Zinc & Trientine \\
\hline Presymptomatic & Zinc & Trientine \\
\hline Pediatric & Zinc & Trientine \\
\hline Pregnant & Zinc & \\
\hline
\end{tabular}

${ }^{a}$ Zinc acetate. Recommended adult dose for all the above indications is $50 \mathrm{mg}$ of elemental zinc three times daily, each dose separated from food and beverages other than water by at least $1 \mathrm{~h}$, and separated from trientine or penicillamine doses by at least $1 \mathrm{~h}$. Pediatric dose for elemental Zinc $1 \mathrm{mg} / \mathrm{kg} / \mathrm{dose}$ (Maximum dose 50mg / dose).

${ }^{\mathrm{b}}$ Trientine is supplied as Syprine and penicillamine as Cuprimine, both manufactured by Merck. Recommended adult dosage for both drugs is $500 \mathrm{mg}$ twice daily, each dose at least $1 / 2 \mathrm{~h}$ before or $2 \mathrm{~h}$ after meals. ${ }^{\mathrm{c}}$ Tetrathiomolybdate is not yet commercially available in India.

\section{Liver transplantation}

If medical therapy fails to suppress the progression of the disease, orthotopic liver transplantation (OLT) is the only alternative treatment. It is indicated for WD patients with acute liver failure when Nazar prognostic score 9 or higher and patients with decompensated cirrhosis that's unresponsive to chelation therapy. Liver transplantations of these individuals can be achieved by a cadaveric donor or living donor transplant, even if the donor is a heterozygous carrier ${ }^{[7]}$.

OLT generally gives excellent results. The one year survival rate and 5 year survival rate in pediatric patients are $90.1 \%$ and $89 \%$ in comparison with a survival of $88.3 \%$ and $86 \%$ for adults in a multi-center observational study over 20 years ${ }^{[8]}$. Liver transplantation is not recommended for patients with neurological and psychiatric symptoms ${ }^{[9]}$.

\section{Diet}

One way to reduce copper absorption is to control the copper content of food. Patients should avoid foods that contain high concentration of copper, such as chocolate, liver, nuts, mushrooms, and shellfish, and using the copper Utensils for cooking food. Wu et al. have found low-copper diet and zinc are effective treatment for the presymptomatic WD patients ${ }^{[11]}$.

\section{The future}

Patients faced with a lifelong need for medication and physicians faced with the results of nonadherence to therapy are the two main arguments. Genetic therapy and hepatocyte transplantation represent future curative treatments for $\mathrm{WD}$, along with currently available liver transplantation. Therapeutic replacement of organs with healthy cells requires disease-specific strategies. As copper toxicosis due to ATP7B deficiency in 
WD produces significant liver injury, disease-specific study of transplanted cell proliferation will offer insights into cell and gene therapy mechanisms. Studies on animal model have showed that cell therapy will correct genetic disorders characterized by organ damage, but suitable mechanisms for inducing transplanted cell proliferation will be critical for therapeutic success ${ }^{[12]}$. However, both cell and liver transplants need immunosuppression to maintain grafted cells. Future use of stem-cells, ex-vivo modification of cells by gene therapy, or better means of inducing immune tolerance may obviate the difficulty of immunosuppresion and provide a cure for WD by cell transplantation.

\section{Conclusion}

In summary, Wilson's disease should be strongly considered as a diagnostic possibility in any child with extra pyramidal neurologic symptoms. Alteration in speech, deterioration in school performance and change in behavior may often be the first symptoms noted. A previous history of jaundice, family history of jaundice or cirrhosis in siblings, increases the index of suspicion for WD exponentially.

\section{References}

[1]. Wilson SAK Progressive lenticular degeneration: a familial nervous disease associated with cirrhosis of the liver. Brain 1912,34: 295-509.

[2]. Huster D: Wilson disease. Best Pract Res Cl Ga 2010, 24(5):531-539.

[3]. Holtzman NA, Gaumnitz BM. Studies on the rate of release and turnover of ceruloplasmin and apoceruloplasmin in rat plasma. $\mathbf{J}$ Biol Chem 1970; 245: 2354-2358.

[4]. Kalra V et al. Wilson's disease: early onset and lessons from a pediatric cohort in India. Indian Pediatr 2000;37: 595-601.

[5]. Sokol RJ. Wilson's Disease and Indian childhood cirrhosis. In: Diseases in Children. Eds. Suchy, Fredrick J. St Louis, Mosby Year Books Inc, 1994; pp 747-772.

[6]. Walshe JM. The Liver in Wilson's Disease (Hepatolenticular Degeneration) In: Diseases of the Liver. Eds Schiff L, Schiff ER, Philadelphia, J.B. Lippincott, 1982; pp 1037-1084.

[7]. Ferenci P, Czlonkowska A, Stremmel W, Houwen R, Rosenberg W, Schilsky M, Jansen P, Moradpour D, Gitlin J: EASL clinical practice guidelines: Wilson's disease. J Hepatol 2012, 56(3):671-685.

[8]. Arnon R, Annunziato R, Schilsky M, Miloh T, Willis A, Sturdevant M, Sakworawich A, Suchy F, Kerkar N: Liver transplantation for children with Wilson disease: comparison of outcomes between children and adults. Clin Transplant 2011, 25(1):E52-E60.

[9]. Murray KF, Carithers RL: AASLD practice guidelines: evaluation of the patient for liver transplantation. Hepatol 2005, 41(6):14071432.

[10]. George J.Brewer. Wilson Disease. Harrison's Principle of Internal Medicine.17 ${ }^{\text {th }}$ Edn, Mc Graw Hill Medical 2008 ; pp $2449-51$.

[11]. Kuruvilla A and Joseph S 'Face of the giant Panda' sign in Wilson's disease: revisited. Neurol India,2000;48: 395-396.

[12]. Hitoshi S, Iwata M,Yoshikawa K. Mid-brain pathology of Wilson Disease: MRI analysis of three cases. J Neurol Neurosurg Psychiatry 1991;54(7):624-26.

[13]. Wu ZY, Lin MT, Murong SX, Wang N: Molecular diagnosis and prophylactic therapy for presymptomatic Chinese patients with Wilson disease. Arch Neurol-Chicago 2003, 60(5):737-741.

[14]. Malhi H, Joseph B, Schilsky ML, Gupta S: Development of cell therapy strategies to overcome copper toxicity in the LEC rat model of Wilson disease. Regen Med 2008, 3(2):165-173.

[15]. Gupta A et al. Molecular pathogenesis of Wilson Disease: haplotype analysis, detection of prevalent mutations and genotypephenotype correlation in Indian patients. Hum Genet 2005;118: 49-57.

[16]. Loudianos $\mathrm{G}$ et al. Improvement of prenatal diagnosis of Wilson disease using microsatellite markers. Prenatal Dian 1994; 14: 999-1002.

[17]. Brewer GJ Penicillamine should not be used as initial therapy in Wilson's disease. Mov Disord 1999;14: 551-554.

[18]. Walshe JM (1982) Treatment of Wilson's disease with trientine (triethylamine tetramine dihydrochloride). Lancet 1982;1: 643-647.

[19]. Brew GJ et al. Treatment of Wilson disease with ammonium tetrathiomolybdate III: Initial therapy in a total of 55 neurologically affected patients and follow up with zinc therapy. Arch Neurol 2003;60: 378-385 